\begin{tabular}{llll} 
Abstarct IDDF2021-ABS-0070 & Table 1 & Baseline characteristics \\
\hline & & $\mathbf{n}$ & Percentage \\
\hline Age (years) & Male & 97 & $62.8 \pm 10.1$ \\
Sex & Female & 9 & $91.5 \%$ \\
& HBV & 80 & $8.5 \%$ \\
Aetiology & HCV & 8 & $75.5 \%$ \\
& HBV+HCV & 1 & $7.5 \%$ \\
& Others & 17 & $0.9 \%$ \\
Serum & Albumin $\geq$ & 69 & $16.0 \%$ \\
albumin (g/1) & 36 & 37 & $65.1 \%$ \\
& Albumin $<36$ & & $34.9 \%$ \\
Total bilirubin & Bilirubin $\leq 17$ & 64 & \\
(mmol/l) & Bilirubin $>17$ & 42 & $60.4 \%$ \\
AFP (ng/ml) & AFP $\leq 400$ & 71 & $39.6 \%$ \\
& AFP $>400$ & 35 & $67 \%$ \\
Tumor size & $\leq 7$ & 68 & $33 \%$ \\
(cm) & $>7$ & 38 & $64.2 \%$ \\
HAP & HAP A & 19 & $35.8 \%$ \\
& HAP B & 41 & $17.9 \%$ \\
& HAP C & 31 & $38.7 \%$ \\
& HAP D & 15 & $29.2 \%$ \\
& & & $14.2 \%$ \\
\hline & & &
\end{tabular}

\begin{tabular}{|c|c|c|c|c|c|c|c|c|c|}
\hline \multicolumn{8}{|c|}{$\begin{array}{l}\text { Abstract IDDF2021-ABS-0070 Table } 2 \\
\text { surviving at the time }\end{array}$} & \multicolumn{2}{|c|}{ Cumulative proportion } \\
\hline \multirow[t]{2}{*}{ HAP } & \multicolumn{2}{|c|}{6 months } & \multicolumn{2}{|c|}{12 months } & \multicolumn{2}{|c|}{24 months } & \multicolumn{2}{|c|}{36 months } & \multirow{2}{*}{$\begin{array}{l}\text { Means for survival } \\
\text { time } \\
\text { (months) }\end{array}$} \\
\hline & $n$ & $(\%)$ & n & $(\%)$ & n & $(\%)$ & $\mathrm{n}$ & $(\%)$ & \\
\hline A & 0 & 100 & 0 & 100 & 1 & 92.3 & 1 & 92.3 & $37.5 \pm 1.2$ \\
\hline B & 1 & 97.4 & 1 & 97.4 & 3 & 88.9 & 5 & 72.5 & $40.8 \pm 2.9$ \\
\hline C & 3 & 88.9 & 5 & 79.9 & 8 & 58.3 & 10 & 31.1 & $24.0 \pm 2.5$ \\
\hline D & 4 & 66.7 & 4 & 66.7 & 5 & 54.2 & 6 & 27.1 & $20.4 \pm 4.1$ \\
\hline
\end{tabular}

(IDDF2021-ABS-0070 Table 1). The median follow-up time was $37.5 \pm 1.2$ months for HAP A, 40.8 \pm 2.9 months for HAP B, 24.0 \pm 2.5 months for HAP C and 20.4 \pm 4.1 months for HAP D. There were no deaths at the first year in HAP A. The cumulative proportion surviving at first years for HAP B, C, D was 97.4; 77.9 and 66.7, respectively. At 36 months, this percentage for HAP A, B, C and D was 92.3; 72.5; 31.1 and 27.1, respectively, (IDDF2021-ABS-0070 Table 2). Survival of all subgroups differed significantly from each other (each $\mathrm{p}$ $<$ 0.05) (IDDF2021-ABS-0070 Figure 2. Kaplan-Meier curve for HAP score). Area under the curve for receiver operating characteristic of HAP score was 0.71 and higher than this of ALBI grade (0.57) (IDDF2021-ABS-0070 Figure 3. ROC curves for HAP score and ALBI grade), indicating a significant performance of HAP score compared with ALBI grade in prognosis of HCC treated with TACE.

Conclusions HAP is a useful score to assist for the management decisions of patients with HCC requiring TACE due to its value in predicting mortality and survival.

\section{IDDF2021-ABS-0071 TUMOR BURDEN SCORE AS A NEW PROGNOSTIC MARKER FOR PATIENTS WITH HEPATOCELLULAR CARCINOMA UNDERGOING TRANSARTERIAL CHEMOEMBOLIZATION}

${ }^{1}$ Shu-Yein Ho*, ${ }^{2}$ Chih-Chieh Ko, ${ }^{2}$ Chien-Wei Su, ${ }^{3}$ Teh-la Huo. ${ }^{1}$ Division of Gastroenterology and Hepatology, Min-Sheng General Hospital, Taoyuan, Taiwan; ${ }^{2}$ Department of Medicine, Taipei Veterans General Hospital, Taipei, Taiwan; ${ }^{3}$ Department of Medical Research, Taipei Veterans General Hospital, Taipei., Taiwan

\subsection{6/gutjnl-2021-IDDF.78}

Background Tumor size and numbers are major determinants of tumor burden in hepatocellular carcinoma (HCC). Patients with HCC undergoing transarterial chemoembolization (TACE) have variables outcomes due to heterogeneity of tumor burden. Recently, tumor burden score (TBS) was proposed to evaluate the extent of tumor involvement. However, the prognostic accuracy of TBS has not been evaluated in HCC. This study aimed to assess its prognostic role in HCC patients undergoing TACE.

Methods A total of 935 treatment-naive HCC patients receiving TACE were retrospectively analyzed. Multivariate Cox proportional hazard model was used to determine independent prognostic predictors.TBS was defined as the distance from the origin of the Cartesian plane and the comparison of two variables: maximum tumor size (X-axis) and number of tumors (Y-axis) so that $\mathrm{TBS}^{2}=(\text { maximum tumor diameter })^{2}+$ (number $^{2}$ of tumors $)^{2}$. Patients have divided accordingly into three groups:high TBS (over 13.74), medium TBS (3.36-13.74), and low TBS (less than 3.36).

Results TBS tended to increase with the increasing size and number of tumors in this study (IDDF2021-ABS-0071 Figure 1. The association of TBS with tumor diameter and numbers). The Cox model showed that serum creatinine $\geq 1.2 \mathrm{mg} /$ dl (hazard ratio(HR):1.296,95\% confidence interval $(\mathrm{CI}): 1.077-1.559, \mathrm{p}=0.006), \quad$ serum $\quad \alpha$-fetoprotein $\geq 400 \mathrm{ng} / \mathrm{dl}$ (HR:2.245,95\%CI:1.905-2.465, p<0.001), vascular invasion (HR:1.870, 95\% CI: 1.520-2.301,p<0.001), medium TBS (HR:1.489,95\%CI:1.206-1.839, p<0.001), high TBS (HR:2.563,95\%CI:1.823-3.602,p<0.001), albumin-bilirubin (ALBI) grade 2-3 (HR:1.521,95\% CI:1.291-1.792,p<0.001),

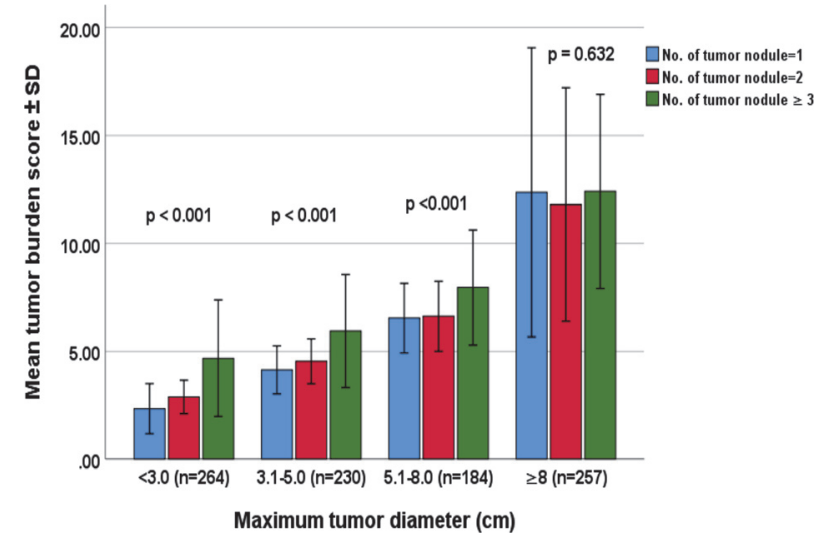

Abstract IDDF2021-ABS-0071 Figure 1 


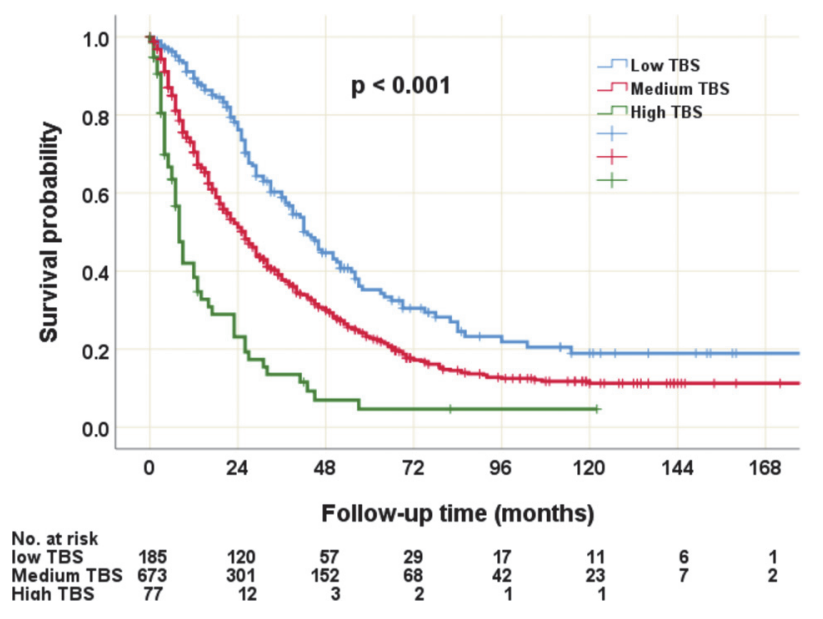

Abstract IDDF2021-ABS-0071 Figure 2

performance status 1(HR:1.362,95\%CI:1.127-1.647,p<0.001) and status 2(HR:1.553,95\%CI: 1.237-1.948,p<0.001) were associated with increased mortality. High TBS had decreased overall survival in comparison with medium TBS and low TBS $(p<0.001$, IDDF2021-ABS-0071 Figure 2. The survival distribution of different TBS in HCC patients undergoing TACE). Patients with high TBS had poor overal survival in Barcelona Clinic Liver Cancer (BCLC) stage B/C and different ALBI grades.

Conclusions TBS is a feasible new prognostic surrogate marker of tumor burden in HCC and can well discriminate survival in patients undergoing TACE across different baseline characteristics.

\section{IDDF2021-ABS-0077 EFFICACY AND SAFETY OF TENOFOVIR ALAFENAMIDE (TAF) VS TENOFOVIR DISOPROXIL FUMARATE (TDF) IN EAST ASIAN CHRONIC HEPATITIS B PATIENTS FOLLOWING 5-YEARS OF TREATMENT}

${ }^{1}$ Grace Lai Hung Wong ${ }^{*},{ }^{2}$ Edward Gane, ${ }^{3}$ Calvin Pan, ${ }^{4}$ Young-Suk Lim, ${ }^{5}$ Scott Fung, ${ }^{6}$ Namiki Izumi, ${ }^{7}$ Mang Ma, ${ }^{8}$ Carol Yee Kwan Chan, ${ }^{9}$ Dr Shalimar, ${ }^{10}$ Maria Buti. ${ }^{1}$ Department of Medicine and Therapeutics, The Chinese University of Hong Kong, Hong Kong: ${ }^{2}$ Department of Medicine, University of Auckland, Auckland, New Zealand; ${ }^{3}$ Division of Gastroenterology and Hepatology, Department of Medicine, NYU Langone Medical Center, NYU School of Medicine, New York, USA; ${ }^{4}$ Department of Gastroenterology, Asan Medical Center, University of UIsan College of Medicine, Sangpa-gu, Seoul, Korea, South; ${ }^{5}$ Toronto Centre for Liver Disease, Toronto General Hospital, University Health Network, Toronto, Ontario, Canada; ${ }^{6}$ Department of Gastroenterology, Musashino Red Cross Hospital, Tokyo, Japan; 'Division of Gastroenterology (Liver Unity), University of Alberta, Edmonton, Canada; ${ }^{8}$ Gilead Sciences, Foster City, CA, USA; ${ }^{9}$ Department of Gastroenterology and Human Nutrition, All India Institute of Medical Sciences, New Delhi, India; ${ }^{10}$ Liver Unit, Vall d'Herbron Hospital, Hospital General Universitario Valle Hebron and Ciberehd, Barcelona, Spain

\subsection{6/gutjnl-2021-IDDF.79}

Background Pivotal studies GS-US-320-0108 (HBeAg-negative) and GS-US-320-0110 (HBeAg-positive), demonstrated non-inferior antiviral efficacy of TAF vs. TDF with superior renal/ bone safety through 5 -years, after up to 3 years of doubleblind (DB) treatment, open-label (OL) TAF was available through year 8 . We analyzed TAF efficacy and safety among patients of Asian Ethnicity in Studies 108/110.
Methods Efficacy was assessed by individual study and included virologic, biochemical, and serologic assessments. Safety data were pooled including estimated GFR (by Cockcroft-Gault method; eGFR $\mathrm{CG}_{\mathrm{G}}$ ) and hip and spine bone mineral density (BMD) changes.

Results Among 1298 patients randomized and treated, 591 (45.5\%) were Asian (TAF $n=410$, while $n=84$ and $n=106$ received TDF-OL-TAF-3 years and TDF-OL-TAF-2 years, respectively. Virologic control was achieved and maintained in patients receiving TAF (95\%) and for TDF-OL-TAF-3 years $(100 \%)$ and TDF-OL-TAF-2 years (98\%). ALT normalization rates were comparable among groups (TAF: 79\%, TDF-OL-TAF-3 years: 80\%; TDF-OL-TAF-2-years: 79\%). HBeAg loss/seroconversion was similar (TAF: 38.6\%/27.4\%, TDF-OL-TAF-3 years: $46.9 \% / 37.5 \%$; TDF-OL-TAF-2-years: 47.1\%/29.4\%). Rates of HBsAg loss/seroconversion were similar in all groups $(\leq 1 \%)$. Rates of Grade $3 / 4$ adverse events (AEs) and AEs leading to discontinuation were low (1.5\%) among all 3 groups. After experiencing declines in $\mathrm{eGFR}_{\mathrm{CG}}$ and in hip/spine BMD over 2 or 3 years of TDF treatment, renal and bone outcomes were improved following the switch to OL TAF.

Conclusions After 5 years of treatment, virologic suppression remained high, and TAF was safe and well-tolerated with improved renal and bone safety among patients of Asian Ethnicity switching from TDF.

\section{IDDF2021-ABS-0078 SWITCHING FROM TENOFOVIR DISOPROXIL FUMARATE (TDF) AND/OR OTHER ORAL ANTIVIRALS (OAVS) TO TENOFOVIR ALAFENAMIDE (TAF) IN VIRALLY SUPPRESSED CHRONIC HEPATITIS B (CHB) PATIENTS WITH MODERATE OR SEVERE RENAL IMPAIRMENT, OR WITH END-STAGE RENAL DISEASE (ESRD)}

${ }^{1}$ Harry LA Janssen* ${ }^{*}$ Pietro Lampertico, ${ }^{3}$ Chien-Hung Chen, ${ }^{4}$ Jeong Heo, ${ }^{5}$ Claire Fournier, ${ }^{6}$ Sang Hoon Ahn, ${ }^{7}$ Tak Yin Owen Tsang, ${ }^{8}$ Carla S Coffin, ${ }^{9}$ Yi-Hsang Huang, ${ }^{10}$ Giulio Marchesini, ${ }^{11}$ Aric Josun Hui, ${ }^{12}$ Magdy Elkhashab, ${ }^{13}$ Sayed-Mohammed Jafri, ${ }^{14} \mathrm{Carol}$ Yee Kwan Chan, ${ }^{14}$ Susanna K Tan, ${ }^{14}$ Yang Zhao, ${ }^{14}$ Vithika Suri, ${ }^{14}$ John F Flaherty, ${ }^{14}$ Anuj Gaggar, ${ }^{14}$ Diana Brainard, ${ }^{15}$ Wan-Long Chuang, ${ }^{16}$ Kosh Agarwal, ${ }^{17}$ Edward Gane, ${ }^{18}$ Young-Suk Lim. ${ }^{1}$ Toronto Centre for Liver Disease, Toronto General Hospital, University Health Network, Toronto, Canada; 'Fondazione IRCCS Ca' Granda Ospedale Maggiore Policlinico, Università di Milano, Milan, Italy; ${ }^{3}$ Kaohsiung Chang Gung Memorial Hospital, Kaohsiung, Taiwan; ${ }^{4}$ Pusan National University School of Medicine, Pusan National University Hospital, Busan, Korea, South; ${ }^{5}$ Department of Medicine, Université de Montréal, Montreal, Québec, Canada; ${ }^{6}$ Yonsei University College of Medicine, Seoul, Korea, South; ${ }^{7}$ Princess Margaret Hospital, Hong Kong; ${ }^{8}$ University of Calgary, Calgary, Alberta, Canada; ${ }^{9}$ Taipei Veterans General Hospital, Taipei, Taiwan; ${ }^{10}$ Azienda Ospedaliero Universitaria di Bologna Policlinico Sant'Orsola-Malpighi, Bologna, Italy; ${ }^{11}$ Alice Ho Miu Ling Nethersole Hospital, Hong Kong; ${ }^{12}$ Toronto Liver Centre, Toronto, Ontario, Canada; ${ }^{13}$ Henry Ford Hospital, Detroit, MI, USA; ${ }^{14}$ Gilead Sciences, Inc., Foster City, CA, USA; ${ }^{15}$ Kaohsiung Medical University Chung-Ho Memorial Hospital, Kaohsiung City, Taiwan; ${ }^{16}$ Kings College Hospital, London, UK; ${ }^{17}$ Auckland Clinical Studies, Grafton, New Zealand; ${ }^{18}$ Asan Medical Center, University of Ulsan College of Medicine, Seoul, Korea, South

\subsection{6/gutjnl-2021-IDDF.80}

Background We have previously shown in renally impaired (RI) CHB patients, including those with ESRD on $\mathrm{HD}$, that switching to TAF from TDF and/or other OAVs maintains high rates of viral suppression with stable bone and renal safety parameters at Week 48. Here we present the final Week 96 results. 